\title{
Incidental COVID-19 on PET/CT imaging
}

\author{
Reshma Amin MD, Leonard Grinblat MD, Mansoor Husain MD
}

Cite as: CMAJ 2020 June 8;192:E631. doi: 10.1503/cmaj.200831; early-released May 22, 2020

A 58-year-old woman with diabetes and morbid obesity (body mass index $44.3 \mathrm{~kg} / \mathrm{m}^{2}$ ) presented to an outpatient facility for routine staging for Hodgkins lymphoma using positron emission tomography-computed tomography (PET/CT) staging. The patient was prescreened outside the clinic doors, and she denied having any symptoms of coronavirus disease 2019 (COVID-19), was afebrile and had no history of travel or contact with anyone with COVID-19. She was injected with fluorodeoxyglucose F 18 ( $\left.{ }^{18} \mathrm{~F}-\mathrm{FDG}\right)$ radiotracer, after which she rested in the supine position in the injection room to allow radiotracer biodistribution, during which the patient was first overheard to have occasional coughing spells.

The PET/CT imaging confirmed the biopsy-proven, stage 2 right pelvic adenopathy (Figure $1 \mathrm{~A}$, white arrow), with only mild ${ }^{18} \mathrm{~F}-\mathrm{FDG}$ activity (SUVmax 2.9). Imaging also showed that the patient had multifocal bilateral peripheral lung opacities (Figures 1C and 1D), with moderate ${ }^{18}$ F-FDG activity (SUVmax 4.5) in the left lower lobe (Figure 1C, white arrow). We did not find any pleural effusions or ${ }^{18} \mathrm{~F}-\mathrm{FDG}$-avid mediastinal adenopathy. One week before presentation, staging diagnostic CT of her chest was clear (Figure 1B).

We immediately notified the referring clinician, and the patient was sent for same-day testing for severe acute respiratory syndrome coronavirus 2 (SARS-CoV-2) with instructions to self-isolate. Two days later, a positive result for reverse transcriptase-polymerase chain reaction (RT-PCR) for SARSCoV-2 was reported; concurrently, the patient had acquired a runny nose, more frequent coughing and fever $\left(38^{\circ} \mathrm{C}\right)$.

Many outpatients have presented for diagnostic imaging after passing prescreening for COVID-19 and have unexpected findings on subsequent chest radiography and $\mathrm{CT},{ }^{1}$ typically multifocal ground-glass opacities or more dense infiltrates. ${ }^{2}$ On PET/CT, pneumonia associated with COVID-19 is ${ }^{18} \mathrm{~F}-\mathrm{FDG}$ avid. ${ }^{3-5}$ Although some researchers have suggested that this modality may be a prognostic indicator, evidence is limited to case reports. ${ }^{3}$

Our patient was admitted to hospital for observation. Her symptoms abated over the course of a week, and she was discharged. Her planned course of pelvic radiation was delayed because of restrictions implemented during the COVID-19 pandemic.

\section{References}

1. Pozzessere C, Rotzinger DC, Ghaye B, et al. Incidentally discovered COVID-19 pneumonia: the role of diagnostic imaging [letter]. Eur Radiol 2020 May 4 [Epub ahead of print]. doi: 10.1007/s00330-020-06914-6.

2. Shi H, Han X, Jiang N, et al. Radiological findings from 81 patients with COVID19 pneumonia in Wuhan, China: a descriptive study. Lancet Infect Dis 2020; 20:425-434.
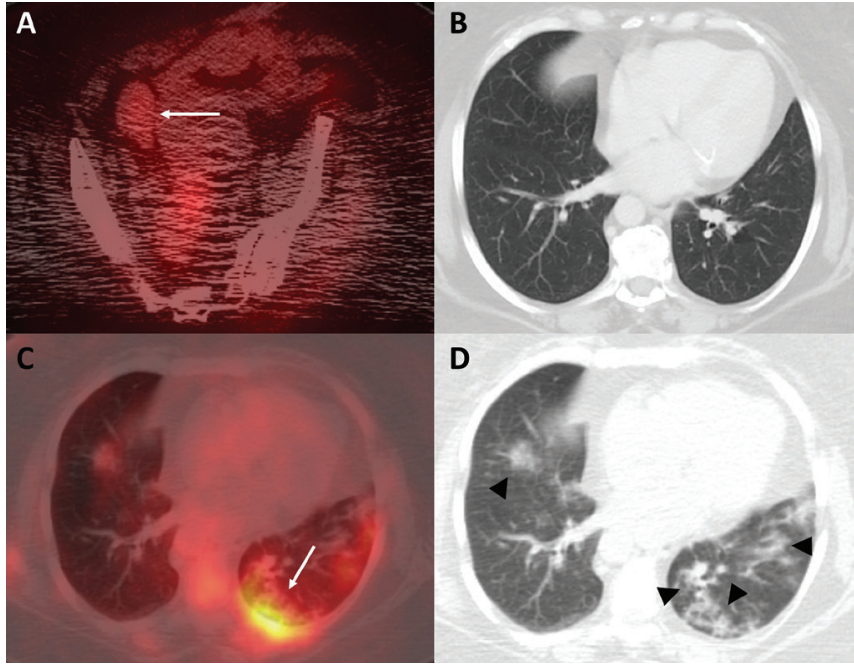

Figure 1: (A) Axial fused positron emission tomography-computed tomography (PET/CT) scan showing uptake of fluorodeoxyglucose F $18\left({ }^{18} \mathrm{~F}-\mathrm{FDG}\right)$ of a lymphoma in the right pelvis (white arrow) of a 58-year-old woman. (B) Axial CT scan of the chest showing clear lung bases on examination performed 1 week before presentation to the clinic. (C) Axial fused PET/CT scan showing multifocal bilateral infiltrates with ${ }^{18} \mathrm{~F}$-FDG activity most notably in the left lower lobe (white arrow) and (D) corresponding CT scan portion from the PET/CT showing multifocal bilateral infiltrates (black arrowheads).

3. Deng Y, Lei L, Chen Y, et al. The potential added value of FDG PET/CT for COVID-19 pneumonia. Eur J Nucl Med Mol Imaging 2020 Mar. 21 [Epub ahead of print]. doi: 10.1007/s00259-020-04767-1.

4. Albano D, Bertagna F, Bertolia M, et al. Incidental findings suggestive of COVID19 in asymptomatic patients undergoing nuclear medicine procedures in a high prevalence region. J Nucl Med 2020;61:632-6.

5. Lütje S, Marinova M, Kütting D, et al. Nuclear medicine in SARS-CoV-2 pandemia: 18F FDG-PET/CT to visualize COVID-19. Nuklearmedizi 2020 Apr. 7. [Epub ahead of print]. doi: 10.1055/a-1152-2341.

Competing interests: Leonard Grinblat and Mansoor Husain are minority shareholders in the privately owned facility (MyHealth Centre, Toronto) where this case originated. No other competing interests were declared.

This article has been peer reviewed.

The authors have obtained patient consent.

Affiliations: SickKids Hospital (Amin); North York General Hospital (Grinblat); MyHealth Centre (Grinblat); University Health Network (Husain), Toronto, Ont.

Acknowledgement: The authors thank Dr. Marc Freeman (MyHealth Centre, Toronto) for assistance with interpretation of positron emission tomography-computed tomography images.

Correspondence to: Leonard Grinblat, l.grinblat@myhealthcentre.ca 